\title{
MODELING SALLEN-KEY AUDIO FILTERS IN THE WAVE DIGITAL DOMAIN
}

\author{
Mattia Verasani, Alberto Bernardini and Augusto Sarti
}

\author{
Dipartimento di Elettronica, Informazione e Bioingegneria \\ Politecnico di Milano, Piazza L. Da Vinci 32, 20133 Milano, Italy \\ Email: mattia.verasani@mail.polimi.it, alberto.bernardini@polimi.it, augusto.sarti@polimi.it
}

\begin{abstract}
Sallen-Key filters are widespread in audio circuits. Therefore, accurate and efficient digital models of such filters are highly desirable in audio Virtual Analog applications. In this paper, we will discuss a possible strategy, based on Wave Digital Filters (WDFs), for implementing all the analog filters described in the historical 1955 manuscript by Sallen and Key. In particular, we will group the eighteen filter models presented by Sallen and Key into nine classes, according to their topological properties. For each class we will describe the corresponding WDF structures. Finally, we will compare the output signals of WDFs to the output signals of the same models implemented in LTSpice.
\end{abstract}

Index Terms - Virtual Analog Modeling, Wave Digital Filters, Audio Filters

\section{INTRODUCTION}

Sallen-Key filters [1] are used pervasively in analog audio circuitry [2]. Therefore, accurate and efficient digital implementations of such filters are extremely useful in audio and music applications based on Virtual Analog (VA) modeling [3]. Some approaches exist in the literature for digitally implementing Sallen-Key filters [4-6], but they are mostly based on discretizing the transfer function, which characterizes the frequency response of the reference filter [4]. Moreover, using these kinds of digital filters, the information about the actual topological structure of the reference analog filter is not preserved in the digital domain. Therefore, such approaches are probably not the best solution for obtaining modular digital models characterized by physically-meaningful parameters. Instead, Wave Digital Filters (WDFs) [7] are suitable for these purposes [8]. Moreover, WDFs are known to be an excellent tool for realizing efficient, accurate and stable digital implementations of linear and Non Linear (NL) reference analog circuits [9. 12], as they turn systems of implicit Kirchhoff equations into a computable graph constituted of blocks, which are connected through input-output relations [13]. For these reasons, they have been widely used in the literature on sound synthesis through physical modeling and interactive VA modeling [14]. However WDFs present also some limitations. In fact, in many cases non-computable Delay-Free-Loops (DFLs) arise also in WDF structures. They may be caused by the presence of multiple non-adaptable NL elements or by complex topologies [15-17]. For accommodating multiple nonlinearities, many methods are under study: approaches based on multi-port NL elements [18-21], iterative methods [8] or dynamic adaptation [22]. For facing topological problems, the approach based on a graph decomposition of the reference circuit by Franken et al. [15] is quite effective, as the circuit can be represented as a computable structure constituted of series, parallel and $R$-type junctions. $R$-type junctions can be implemented using one of the three methods presented in 16 17 23].

In order to effectively implement Sallen-Key filters in the Wave Digital (WD) domain, besides having to accommodate complex topologies, it is also essential to rely on efficient and accurate operational amplifier (op-amp) models. In the literature on WDFs there are only few works in which circuits containing op-amps are discussed; [24], [25] and [26]. In particular, in [26] a Modified Nodal Analysis (MNA)-based method is described for accommodating arbitrarily complex linear circuits containing op-amps, along with two possible op-amp models; one ideal model based on nullors and one more complex linear macromodel containing some linear nonidealities. In the light of the approach described in [17 26], in this paper, we will analyze all the Sallen-Key filters and we will derive their WD implementations, using a simplified, though accurate, real op-amp macromodel. The paper is organized as follows. In Section 2 we will group the eighteen Sallen-Key filters presented in [1] into nine classes, according to their topological properties. In Section 3 we will briefly describe how to proceed for modeling a generic circuit with complex topologies in the WD domain. In Section 4 for each one of the nine classes defined in Section 2 we will show how to build the corresponding WDF model, following the approach presented in [17, 26]. For modeling op-amps, we will use a static small-signal macromodel, which is simpler w.r.t. the linear macromodel presented in [26]. In Section 5, we will test the derived WDF models through some examples of applications and we will show the resulting output signals are nearly identical to the corresponding LTSpice implementations. Finally, Section 6 concludes this paper.

\section{SALLEN-KEY FILTERS CLASSIFICATION}

Sallen-Key filters are second order electronic active filters containing op-amps, introduced in the 1955 manuscript [1] by Sallen and Key. They have been widely used, not only in audio circuits [27], for implementing low-pass, high-pass and band-pass frequency responses. In the original work [1], such filters involved only resistors and capacitors. In [1] a "catalog of second order active networks" with eighteen numbered filters is presented. Analyzing those filters we identified nine recurring topological patterns, which are shown in Fig. 11 The white rectangular blocks indicate generic impedances $Z_{h}$ (with $h$ an integer), which could also be combinations of elements in series or parallel. The numbered filters in the Sallen and Key catalog are classified in Fig. 1 as follows: (a) refers to filters 1, 3 and 9, (b) refers to 5 and 7 , (c) refers to 10 and 13 , (d) refers to 11 and 14 , (e) refers to 16 and 17, (f) refers to 12 and 15, (g) refers to 6 and 8 , (h) refers to 2 and 4 , while (i) refers to 18 . As it is evident from Fig. 1, in the classification process, we considered also the op-amp 


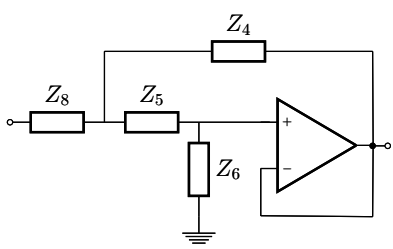

(a)

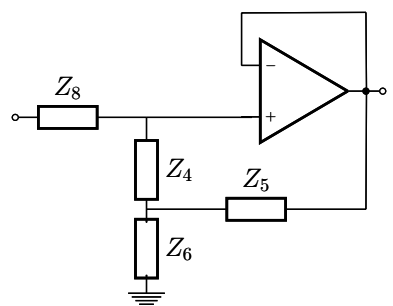

(c)

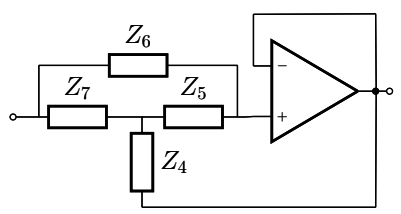

(e)

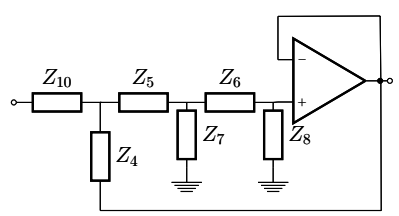

(g)

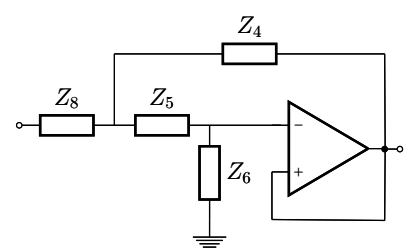

(b)

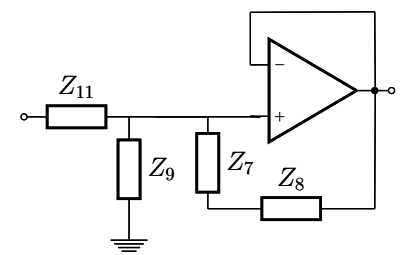

(d)

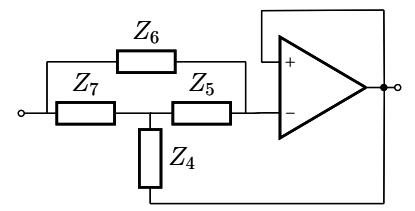

(f)

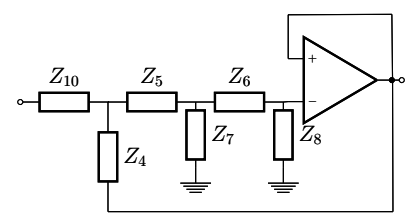

(h)

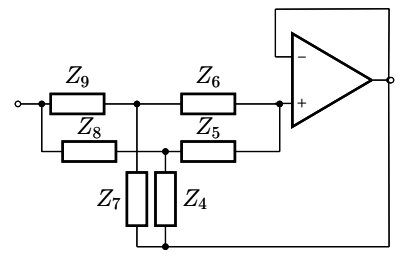

(i)

Fig. 1. Nine classes of Sallen-Key filters with different topologies.

polarity as a discriminative property. Therefore, the circuits pairs (a)-(b), (e)-(f) and (g)-(h) share the same topological structure, but the positive and negative input terminals of the op-amp are swapped.

\section{WDF MODELING OF CIRCUITS}

WDFs are derived performing a lumped discretization of a reference analog circuit and applying a port-wise linear transformation. If the reference circuit is a $N$-port network, the vectors of port voltages $\mathbf{v}=\left[v_{1}, \ldots, v_{N}\right]^{t}$ and port currents $\mathbf{i}=\left[i_{1}, \ldots, i_{N}\right]^{t}$ are mapped into the vectors of incident waves $\mathbf{a}=\left[a_{1}, \ldots a_{N}\right]^{t}$ and reflected waves $\mathbf{b}=\left[b_{1} \ldots b_{N}\right]^{t}$, as follows

$$
\mathbf{a}=\mathbf{v}+\mathbf{R i} \quad, \quad \mathbf{b}=\mathbf{v}-\mathbf{R i}
$$

where $\mathbf{R}=\operatorname{diag}\left[R_{01}, \ldots R_{0 N}\right]$ is the diagonal matrix of the port free parameters, called reference port resistances. Often it is also useful to define the port admittance matrix $\mathbf{G}=\mathbf{R}^{-1}=$ $\operatorname{diag}\left[G_{01}, \ldots G_{0 N}\right]$. In the WD domain, each element becomes a block characterized by an input-output relation [7] and the topological connections between the elements are implemented using multi-port junctions, called adaptors [28]. How to accommodate the most spread linear circuit elements (e.g. real sources, resistors, capacitors) and traditional series and parallel adaptors is extensively explained in [7, 28]. In order to solve more complex connections than series and parallel, the method by Franken et al. [15] is used for turning the reference circuit graph into a $S P Q R$-tree structure, in order to identify the $S$-, $P$-, $Q$ - and $R$-type portions. Then, the corresponding adaptors of the $R$-type nodes should be derived using one of the approaches described in the three works [23], [16] and $[17]$. As pointed out in $[25]$ in Fig. 8, where a circuit with a simple op-amp model is studied, in some cases a replacement graph [15] must be added to the circuit graph representation in order to embed multi-port active elements into $R$-type nodes and avoid DFLs. Therefore, especially in some particular cases where controlled sources are involved, the implementation of hybrid active junctions, which embody both topological connections and active multi-port elements, seems unavoidable for ensuring computability. The MNA-based [29] method presented in [17, 25, 26 30] allows us to derive the scattering matrix $\mathbf{S}$ of such active junctions as follows

$$
\mathbf{S}=\mathbf{I}+\left[\begin{array}{lll}
\mathbf{0} & \mathbf{R} & \mathbf{0}
\end{array}\right] \mathbf{X}^{-1}\left[\begin{array}{lll}
\mathbf{0} & \mathbf{I} & \mathbf{0}
\end{array}\right]^{t} \text { with } \mathbf{b}=\mathbf{S a},
$$

where $\mathbf{I}$ is the identity matrix, $\mathbf{0}$ is a matrix of zeros with the appropriate size and $\mathbf{X}$ is the so called MNA matrix. The MNA analysis is applied to the circuit corresponding to the $R$-type components of the $S P Q R$-tree to which ports are attached Thévenin equivalents. The ideal voltage sources of the Thévenin equivalents are ordered in the vector $\mathbf{e}=\left[e_{1}, \ldots, e_{N}\right]^{t}$, while the corresponding branch currents are $\mathbf{j}_{1}=\left[j_{1}, \ldots, j_{N}\right]^{t}$. Applying the analogy, which is described in [17], using the concept of instantaneous Thévenin equivalents, and also explained in [18], under the name of Kirchhoff Domain Analogy, we set $\mathbf{e}=\mathbf{a}, \mathbf{j}=\left[\mathbf{j}_{\mathbf{1}}^{\mathrm{t}}, \mathbf{j}_{\mathbf{2}}^{\mathrm{t}}\right]^{t}=\left[-\mathbf{i}^{t}, \mathbf{j}_{\mathbf{2}}\right]^{t}$, where $\mathbf{j}_{\mathbf{2}}$ are other branch currents not passing through the instantaneous Thévenin equivalents (e.g. through Voltage Controlled Voltage Source (VCVS)), and we set the port resistances equal to the Thévenin equivalents series resistances. More precisely, $\mathbf{X}$ satisfies the following system of equations

$$
\mathbf{X}\left[\begin{array}{c}
\mathbf{v}_{\mathbf{n}} \\
\mathbf{j}
\end{array}\right]=\left[\begin{array}{cc}
\mathbf{Y} & \mathbf{A} \\
\mathbf{B} & \mathbf{D}
\end{array}\right]\left[\begin{array}{c}
\mathbf{v}_{\mathbf{n}} \\
\mathbf{j}
\end{array}\right]=\left[\begin{array}{c}
\mathbf{i}_{\mathbf{s}} \\
\mathbf{e}
\end{array}\right]
$$

where the $\mathbf{X}$ partitions $\mathbf{Y}, \mathbf{A}, \mathbf{B}$, and $\mathbf{D}$ define the relation among $\mathbf{e}, \mathbf{j}$, the node voltages $\mathbf{v}_{\mathbf{n}}$ and the current source values $\mathbf{i}_{\mathbf{s}}$, which in our context are always zero. Once all the needed WD elements and adaptors are derived, systematic methods for initializing and implementing generic WDF structures could be used, as explained in [13].

\section{SALLEN-KEY FILTERS IN THE WD DOMAIN}

In this Section, we describe how to apply the WDF approach resumed in Section 3 in order to implement all the Sallen-Key topologies. The MNA method, described in Section 3 , would work independently on the used op-amp macromodel. However, in this paper, we will describe op-amps with the linear small-signal static macromodel in Fig. 2. $R_{\text {in }}$ is the finite, typically very high, input resistance. $R_{\text {out }}$ is the non-zero, typically very low, output resistance. $A_{0}$ is the finite open-loop gain. $R_{\text {in }}, R_{\text {out }}$ and $A_{0}$ are usually specified in the reference op-amp datasheet. The VCVS is controlled by 


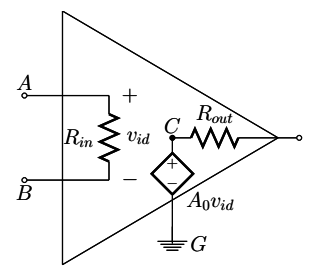

Fig. 2. Op-amp linear static small signal macromodel.

the signal $A_{0} v_{i d}$. The nodes $A, B, C$ and $G$ indicate the terminals of the op-amp macromodel.

Let us now consider the nine models shown in Fig. 1 For each filter in Fig. 1 we connect a real voltage source $V_{i n}$ to the input port and a load resistance $R_{\text {load }}$ to the output port. In order to derive the corresponding WDF structures, we have firstly to build the relative graphs. Then we find the split components of the $S P Q R$ tree structure, forcing the nodes $A, B, C$ and $G$ to be part of a $R$-type node, using a replacement graph, as explained in $|15|$ and as shown also in Fig. 8 in [25]. A graph representation of the circuits in Fig. 1 is shown in Fig. 3, the fictitious edges of the replacement graphs are colored in gray. We labeled the edges of the graphs using the $Z_{h}$ impedances subscripts (with $h$ an integer) shown in Fig. 11. while we always assign the label 1 to the element $R_{i n}, 2$ to $R_{\text {out }}$ and 3 to $R_{\text {load }}$. We named the different $R$-types using different subscripts. From the circuit graph representation, it is now easy to build the scheme of the corresponding WDF structures. As an example, in Fig. 4 we show the WDF structure resulting from the $S P Q R$-tree in Fig. 3 d. In order to find the scattering matrix of the $R$ node $R_{\tau}$, we perform the MNA analysis of the circuit in Fig. 5 i.e. the $R$-node circuit with instantaneous Thévenin equivalents attached at each port. The MNA matrix relative to $R_{\tau}$ is called $\mathbf{X}_{\tau}$ and, according to Fig. 5 if we set $\mathbf{v}_{\mathbf{n}}=\left[v_{m}, v_{n}, v_{o}, v_{t}, v_{A}, v_{C}, v_{B}\right]^{t}$, $\mathbf{j}=\left[-i_{5},-i_{4},-i_{2},-i_{3}, j_{V C V S}\right]^{t}, \mathbf{e}=\left[a_{5}, a_{4}, a_{2}, a_{3}\right]^{t}$, the corresponding $\mathbf{X}_{\tau}$ partition matrices are: $\mathbf{D}_{\tau}=\mathbf{0}$,

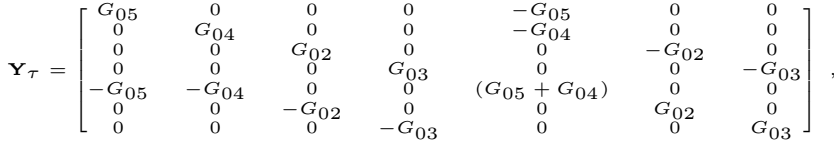$$
\mathbf{A}_{\tau}=\left[\begin{array}{ccccc}
1 & 0 & 0 & 0 & 0 \\
0 & 1 & 0 & 0 & 0 \\
0 & 0 & 1 & 0 & 0 \\
0 & 0 & 0 & 1 & 0 \\
0 & 0 & 0 & 0 & 0 \\
0 & 0 & 0 & 0 & 1 \\
0 & -1 & -1 & 0 & 0
\end{array}\right] \quad, \quad \mathbf{B}_{\tau}=\left[\begin{array}{ccccccc}
1 & 0 & 0 & 0 & 0 & 0 & 0 \\
0 & 1 & 0 & 0 & 0 & 0 & -1 \\
0 & 0 & 1 & 0 & 0 & 0 & -1 \\
0 & 0 & 0 & 1 & 0 & 0 & 0 \\
0 & 0 & 0 & 0 & -A_{0} & 1 & A_{0}
\end{array}\right]
$$

Finally we use equation (2) for finding $S_{\tau}$. All the other scattering matrices of the $R$-type nodes in Fig. 3 can be derived similarly.

As in traditional adaptors, also one port of such $R$-type nodes can be made reflection free. For instance, in our example, we adapt the left port of $R_{\tau}$ by setting $G_{05}=\frac{G_{04} G_{02}+G_{04} G_{03}}{G_{04}+G_{02}+G_{03}+A_{0} G_{02}}$.

\section{EXAMPLES OF APPLICATIONS}

In this Section, we analyze the behavior of the derived WD SallenKey models, feeding them with a linear sinusoidal up-chirp signal with $f_{1}=20 \mathrm{~Hz}$ and $f_{2}=2000 \mathrm{~Hz}$ as starting and ending frequencies. The amplitude of that signal is $3 \mathrm{~V}$. A zoomed portion of the up-chirp is shown at the top of Fig. 6. The output signals are compared to the outputs of the corresponding LTSpice simulations.

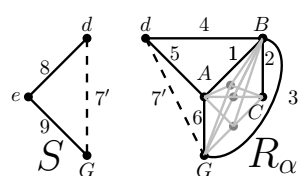

(a)
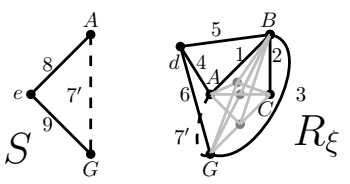

(c)

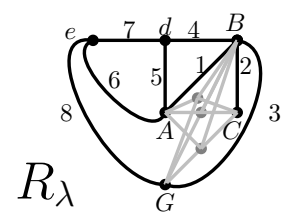

(e)

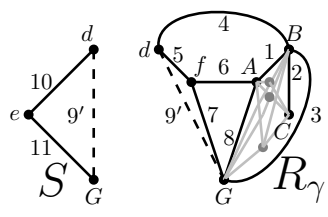

(g)

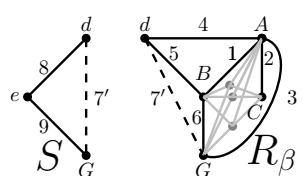

(b)

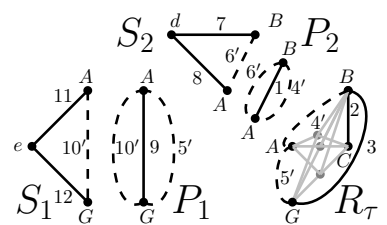

(d)

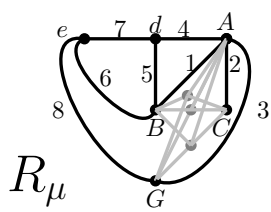

(f)
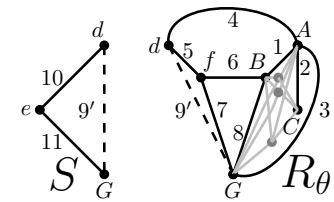

(h)

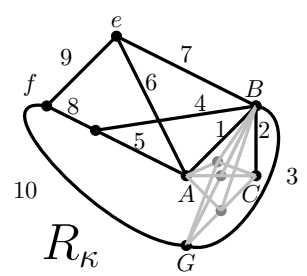

(i)

Fig. 3. Finding split components of the nine filters in Fig. 1

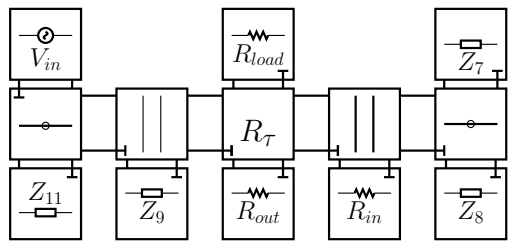

Fig. 4. WDF structure relative to the graph in Fig. 3 d.

We use a LTSpice model of the LM324 op-amp, which is very common in some audio synthesizers, such as the Korg Monotron [31]. The needed parameters are taken from the LM324 datasheet [32]. The LM324 LTSpice model is employed in all the circuit simulations. According to the LM324 datasheet, we set the small-signal static macromodel parameters to $R_{\text {in }}=10^{6} \Omega, R_{\text {out }}=10 \Omega$ and $A_{0}=10^{5}$. We implement variants of the filters in Fig. 1 Here we show the results of five significant Sallen-Key configurations. The sampling rate is $F_{s}=48 \mathrm{kHz}$. For the sake of brevity, we define 


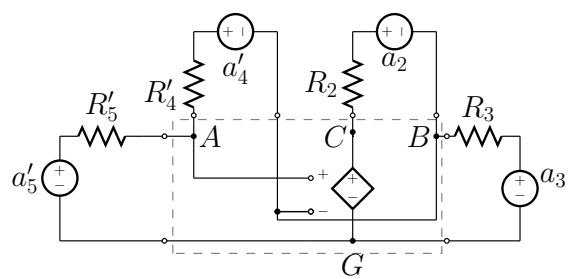

Fig. 5. Kirchhoff Domain Analogy Circuit for MNA Analysis (referred to Fig. 3 d).

the capacitance parameter $C=0.1 \mathrm{nF}$ and the resistance parameter $R=100 \mathrm{k} \Omega$. In the corresponding figures captions, we will specify the actual filter impedances values used in each simulation. As far as the following plots are concerned, we define the $V_{\text {load }}$ LTSpice signal as $\bar{V}_{\text {load }}$ and the same signal taken from the WDF structure as $\hat{V}_{\text {load }}$. In each plot we compare $\hat{V}_{\text {load }}$ to $\bar{V}_{\text {load }}$ and we show the squared error signal $V_{\text {error }}^{2}=\left(\hat{V}_{\text {load }}-\bar{V}_{\text {load }}\right)^{2}$.
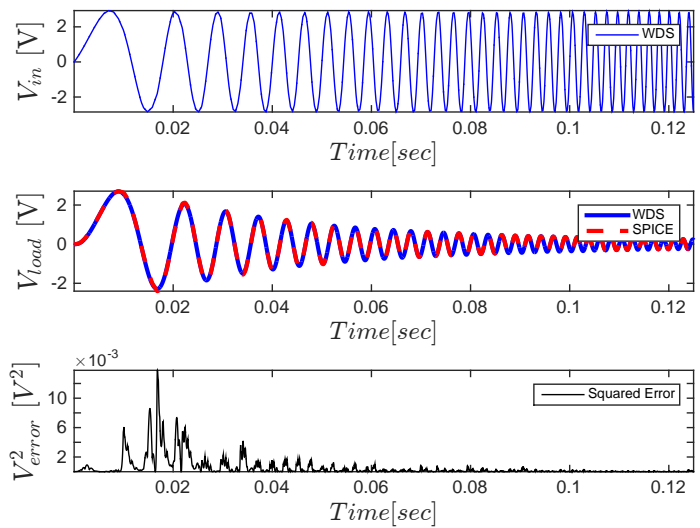

Fig. 6. Top: Up-chirp signal $V_{i n}$ used for all the simulations. Center: $V_{\text {load }}$ signal WDF model vs LTSpice. The reference model is the one in Fig. 1 1 a; in particular $Z_{4}=1 / s C, Z_{5}=R, Z_{6}=1 / s C$ and $Z_{8}=R$. Bottom: Squared Error signal WDF model vs LTSpice.
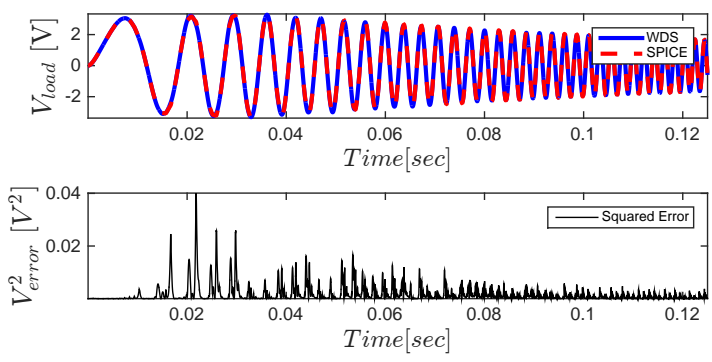

Fig. 7. $V_{\text {load }}$ WDF model vs LTSpice. The reference Sallen-Key model is the one in Fig. 1] c; in particular $Z_{4}=1 / s C, Z_{5}=R$, $Z_{6}=1 / s C$ and $Z_{8}=R$.

\section{CONCLUSION AND FUTURE WORK}

In this paper, we analyzed the eighteen RC active filters proposed by Sallen and Key in their original manuscript and we divided them
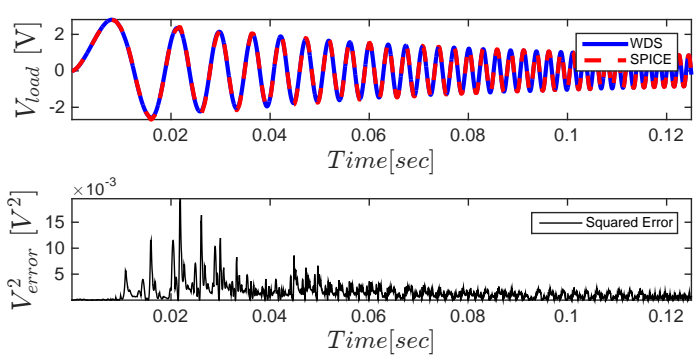

Fig. 8. $V_{\text {load }}$ WDF model vs LTSpice. The reference Sallen-Key model is the one in Fig. $11 \mathrm{~d}$; in particular $Z_{7}=1 / s C, Z_{8}=R$, $Z_{9}=1 / s C$ and $Z_{11}=R$.
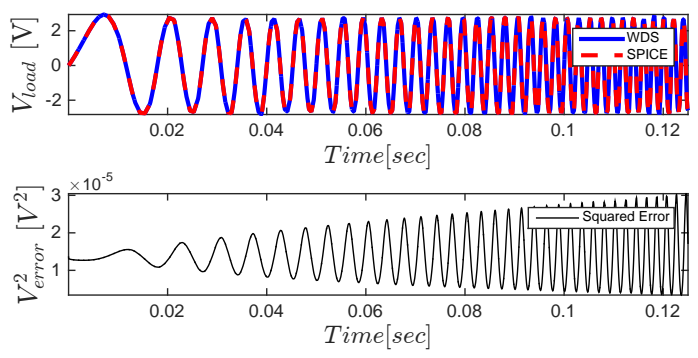

Fig. 9. $V_{\text {load }}$ WDF model vs LTSpice. The reference Sallen-Key model is the one in Fig. 1 e; in particular $Z_{4}=1 / s C, Z_{5}=R$, $Z_{6}=1 / s C$ and $Z_{7}=R$.
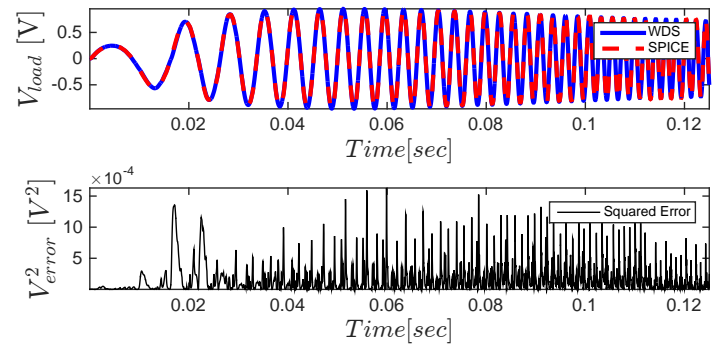

Fig. 10. $V_{\text {load }}$ WDF model vs LTSpice. The reference Sallen-Key model is the one in Fig. $1 \mathrm{~g}$; in particular $Z_{4}=R / 3, Z_{5}=R / 3$, $Z_{6}=1 / s C, Z_{7}=1 / s C, Z_{8}=R$ and $Z_{10}=R / 3$.

into nine possible classes, according to their topological properties. Then we showed how to develop the parametric WDF model for each class. Finally, we showed that the WDF output signals greatly match the corresponding LTSpice simulation results, even though some small errors are present. Such errors are not necessarily produced entirely by WDFs. In fact, they might be partially due to the numerical inaccuracies of the iterative solvers employed by LTSpice and/or the re-sampling process (from non-uniform sampling to uniform sampling) performed on LTSpice output signals. The presented models are highly modular, independent from the actual filter impedances and they preserve the topological properties of the reference analog circuits. Moreover, as all traditional WDFs, they are suitable to be used for interactive VA applications [3. 14].

As far as future work is concerned, we are planning to apply the same methodology in order to derive WD models of more complex filter topologies involving op-amps, such as biquad filters. 


\section{REFERENCES}

[1] R. P. Sallen and E. L. Key, "A practical method of designing rc active filters," IRE Trans. Circuit Theory, vol. 2, pp. 74-85, 1955.

[2] B. Klein, Electronic Music Circuits, 1st ed. Howard W. Sams and Co., Inc., 1982.

[3] G. De Sanctis and A. Sarti, "Virtual analog modeling in the wave-digital domain," IEEE Trans. Audio, Speech, Language Process., vol. 18, pp. 715-727, May 2010.

[4] W. Pirkle, Designing Software Synthesizer Plug-ins in C++: For RackAFX, VST3, and Audio Units, 1st ed. Focal Press, Taylor and Francis Group, 2015.

[5] W. Pirkle, "Modeling the korg35 lowpass and highpass filters," in Audio Engineering Society Convention 135. New York, NY: Audio Engineering Society, Oct. 17 - Oct. 202013.

[6] W. Pirkle, "Novel hybrid virtual analog filters based on the sallen-key architecture," in Audio Engineering Society Convention 137. Los Angeles, CA: Audio Engineering Society, Oct. 9 - Oct. 122014.

[7] A. Fettweis, "Wave digital filters: Theory and practice," Proc. of the IEEE, vol. 74, pp. 270-327, Feb. 1986.

[8] T. Schwerdtfeger and A. Kummert, "Newton's method for modularity-preserving multidimensional wave digital filters," in IEEE 9th Int. Workshop on Multidimensional (nD) Systems (nDS), Vila Real, Portugal, Sept. 7-9 2015, pp. 1-6.

[9] A. Fettweis, "Pseudo-passivity, sensitivity, and stability of wave digital filters," IEEE Trans. Circuit Theory, vol. 19, pp. 668-673, Nov. 1972.

[10] K. Meerkötter and R. Scholz, "Digital simulation of nonlinear circuits by wave digital filter principles," in IEEE Int. Symp. Circuits Syst., vol. 1, June 1989, pp. 720-723.

[11] A. Sarti and G. De Poli, "Toward nonlinear wave digital filters," IEEE Trans. Signal Process., vol. 47, pp. 1654-1668, June 1999.

[12] K. J. Werner, V. Nangia, A. Bernardini, J. O. Smith, and A. Sarti, "An improved and generalized diode clipper model for wave digital filters," in Proc. 139th Conv. Audio Eng. Soc. (AES), New York, NY, Oct. 29 - Nov. 12015.

[13] A. Sarti and G. De Sanctis, "Systematic methods for the implementation of nonlinear wave-digital structures," IEEE Trans. Circuits Syst. I, Reg. Papers, vol. 56, pp. 460-472, Feb. 2009.

[14] F. Pedersini, A. Sarti, and S. Tubaro, "Object-based sound synthesis for virtual environments using musical acoustics," IEEE Signal Process. Mag., vol. 17, pp. 37-51, Nov. 2000.

[15] D. Fränken, J. Ochs, and K. Ochs, "Generation of wave digital structures for connection networks containing multiport elements," IEEE Trans. Circuits and Systems I: Regular Papers, vol. 52, pp. 586-596, March 2005.

[16] K. Meerkötter and D. Franken, "Digital realization of connection networks by voltage-wave 2-port adapters," $A E U$ - International Journal of Electronics and Communications, vol. 50(6), pp. 362-367, 1996.

[17] K. J. Werner, V. Nangia, J. O. Smith, and J. S. Abel, "A general and explicit formulation for wave digital filters with multiple/multiport nonlinearities and complicated topologies," in Proc. IEEE Workshop on Applicat. of Signal Process. to Audio and Acoust. (WASPAA), New Paltz, NY, Oct. 18-21 2015.
[18] A. Bernardini, K. J. Werner, A. Sarti, and J. O. Smith, "Modeling nonlinear wave digital elements using the Lambert function," IEEE Trans. Circuits and Systems I: Regular Papers, vol. 63, pp. 1231-1242, August 2016.

[19] A. Bernardini, K. J. Werner, A. Sarti, and J. O. Smith, "Multiport nonlinearities in wave digital structures," in Proc. IEEE Int. Symp. Signals Circuits Syst. (ISSCS 2015), Iasi, Romania, July 9-10 2015.

[20] A. Bernardini, K. J. Werner, A. Sarti, and J. O. Smith, "Modeling a class of multi-port nonlinearities in wave digital structures," in Proc. European Signal Process. Conf. (EUSIPCO), Nice, France, Aug. 31 - Sept. 4 2015, pp. 669-673.

[21] S. D'Angelo, J. Pakarinen, and V. Välimäki, "New family of wave-digital triode models," IEEE Trans. Audio, Speech, Language Process., vol. 21, pp. 313-321, Feb. 2013.

[22] A. Bernardini and A. Sarti, "Dynamic adaptation of instantaneous nonlinear bipoles in wave digital networks," in Proc. European Signal Process. Conf. (EUSIPCO), Budapest, Hungary, Aug. 29 - Sept. 22016.

[23] G. O. Martens and K. Meerkötter, "On n-port adaptors for wave digital filters with application to a bridged-tee filter," in Proc IEEE Int. Symp. Circuits Syst., Munich, Germany, Apr. 1976, p. 514517.

[24] R. C. D. Paiva, S. D’Angelo, J. Pakarinen, and V. Välimäki, "Emulation of operational amplifiers and diodes in audio distortion circuits," IEEE Trans. Circuits Syst. II, Exp. Briefs, vol. 59, pp. 688-692, Oct. 2012.

[25] K. J. Werner, J. O. Smith, and J. S. Abel, "Wave digital filters adaptors for arbitrary topologies and multiport linear elements," in Proc. 18th Conf. Digital Audio Effects (DAFx-15), Trondheim, Norway, Nov. 30 - Dec. 32015.

[26] K. J. Werner, W. R. Dunkel, M. Rest, M. J. Olsen, and J. O. Smith, "Wave digital filter modeling of circuits with operational amplifiers," in Proc. European Signal Process. Conf. (EUSIPCO), Budapest, Hungary, Aug. 29- Sept. 2016.

[27] B. Razavi, Fundamentals of microelectronics, 2nd ed. John Wiley and Sons Inc., 2009.

[28] A. Fettweis and K. Meerkötter, "On adaptors for wave digital filters," IEEE Trans. Acoust., Speech, Signal Process., vol. 23, pp. 516-525, Mar. 1975.

[29] C. Ho, A. Ruehli, and P. Brennan, "The modified nodal approach to network analysis," IEEE Transactions on Circuits and Systems, vol. 22, pp. 504-509, 1975.

[30] K. J. Werner, V. Nangia, J. O. Smith, and J. S. Abel, "Resolving wave digital filters with multiple/multiport nonlinearities," in Proc. 18th Conf. Digital Audio Effects (DAFx-15), Trondheim, Norway, Nov. 30 - Dec. 32015.

[31] KORG, Schematic for public release: monotron DELAY, 2012.

[32] Texas Instruments, LMx24-N, LM2902-N Low-Power, QuadOperational Amplifiers, 2015. 